\title{
Nacionalismo, Patriotismo e Essencialismo na Construção da Identidade Nacional Brasileira
}

\author{
Eldo Lima Leite*, 1 \\ Orcid.org/0000-0002-5152-8353 \\ Andreza Silene Silva Ferreira ${ }^{1}$ \\ Orcid.org/0000-0001-9068-0262 \\ José Roniere Morais Batista ${ }^{2}$ \\ Orcid.org/0000-0002-9055-7544 \\ José Luiz Álvaro Estramiana ${ }^{3}$ \\ Orcid.org/0000-0002-3017-0305 \\ Ana Raquel Rosas Torres ${ }^{1}$ \\ Orcid.org/0000-0002-3161-0309 \\ ${ }^{1}$ Universidade Federal da Paraíba, João Pessoa, PB, Brasil \\ ${ }^{2}$ Universidade Federal de Campina Grande, Campina Grande, PB, Brasil \\ ${ }^{3}$ Universidade Complutense de Madrid, Madrid, Espanha
}

\section{Resumo}

Este trabalho objetivou testar a hipótese de que o nacionalismo e o patriotismo são elementos definidores do grau de identificação com a nação brasileira e que o essencialismo tem um papel mediador na relação entre o patriotismo, o nacionalismo e a identidade nacional. Participaram deste estudo 229 estudantes universitários com idades média de 20 anos, $D P=3,6$; sendo $28,8 \%$ do sexo masculino e $71,2 \%$ do sexo feminino. Os resultados demonstram que a identidade nacional brasileira é um construto multifacetado e que pode ser explicada a partir de fatores como patriotismo, nacionalismo e essencialismo. Referente ao papel destas variáveis no modelo, corroboramos nossa hipótese de que o essencialismo funciona como uma variável mediadora da relação entre patriotismo, nacionalismo e identidade nacional. Apesar do essencialismo explicar a identidade nacional, o seu papel não é constitutivo, mas processual. Seria o pensamento essencialista quem explicaria a parcela da identidade nacional percebida em termos de atributos homogêneos. Neste sentido, ele funciona como um viés cognitivo através do qual os indivíduos percebem e organizam, em termos categóricos, o seu grupo nacional. Estes resultados nos ajudam a entender o papel que o nacionalismo, o patriotismo e o essencialismo têm na construção da identidade nacional brasileira.

Palavras-chave: Identidade nacional, Patriotismo, Nacionalismo, Essencialismo.

* Endereço para correspondência: Rua Comerciante Alfredo Ferreira da Rocha, $\mathrm{n}^{\mathrm{o}}$ 1745, Bairro Mangabeira, João Pessoa, PB, Brasil 58055-541. Fone: +55 83 99647-4692. E-mail: eldolima@gmail.com Esta pesquisa contou com apoio financiero do Conselho Nacional de Desenvolvimento Científico e Tecnológico (CNPq). 


\title{
Nationalism, Patriotism, and Essentialism in the Construction of Brazilian National Identity
}

\begin{abstract}
The main aim of this study was to test the hypothesis that nationalism and patriotism are fundamental elements in the degree of identification with the Brazilian nation and that essentialism has a mediating role in the relationship between nationalism, patriotism and national identity. A total of 229 university students took part in this study, with a mean age of 20 years; $28 \%$ were men and $71.2 \%$ women. The results of this study show that Brazilian national identity is a multifaceted construct that can be explained by taking into account factors such as nationalism, patriotism, and essentialism. In relation to the role played by these variables in the model, our hypothesis that essentialism acts as a mediating variable in the relationship between patriotism, nationalism, and national identity was confirmed. Even though essentialism explains national identity, it does not have a constitutive role, because of its process-based nature. It explains perceived national identity in terms of homogeneous attributes. These results help us in understanding the role of nationalism, patriotism, and essentialism in the construction of the Brazilian national identity.
\end{abstract}

Keywords: National identity, Patriotism, Nationalism, Essentialism.

\section{Nacionalismo, Patriotismo y Esencialismo en la Construcción de la Identidad Nacional Brasileña}

\section{Resumen}

Este trabajo tuvo como objetivo comprobar la hipótesis de que el nacionalismo y el patriotismo son elementos definidores del grado de identificación con la nación brasileña y que el esencialismo tiene un papel mediador en la relación entre nacionalismo, patriotismo e identidad nacional. Participaron en este estudio 229 estudiantes universitarios, con una edad media de 20 años, $D E=3$; de los cuales el $28 \%$ eran hombres y el 71,2\% mujeres. Los resultados demuestran que la identidad nacional brasileña es un constructo multifacético que puede ser explicado a partir de factores como el nacionalismo, el patriotismo y el esencialismo. En relación al papel de estas variables en el modelo, corroboramos nuestra hipótesis de que el esencialismo actúa como una variable mediadora de la relación entre patriotismo, nacionalismo e identidad nacional. A pesar de que el esencialismo explica la identidad nacional, su papel no es carácter constitutivo sino procesual. El pensamiento esencialista explica la identidad nacional percibida en términos de atributos homogéneos. En este sentido, actúa como un sesgo cognitivo a través del cual los individuos perciben y organizan, en términos categóricos, su grupo nacional. Estos resultados nos ayudan a entender el papel que el nacionalismo, el patriotismo y el esencialismo juegan en la construcción de la identidad nacional brasileña.

Palabras clave: Identidad nacional, Patriotismo, Nacionalismo, Esencialismo.

O estudo da identidade nacional brasileira tem sido considerado um tema de crescente interesse, devido a maior representatividade do Brasil no cenário econômico mundial (Cavusgil \& Kardes, 2013). Ela é considerada como um desafio social (Sobral \& Vala, 2010; Terlouw, 2013), como um dos fenômenos de maior valor político dos nossos tempos (Anderson, 1991).
No entanto, os psicólogos sociais têm dado pouca importância à questão do porquê que os indivíduos se sentem, se identificam, lutam e morrem por seus países (Reicher \& Hopkins, 2001). Na área da psicologia social, poucos artigos foram publicados sobre a identidade nacional brasileira (Lima, Torres, \& Techio, 2016). Em um levantamento realizado no Periódicos 
Capes usando as palavras-chave "brazilian $n a$ tional identity", selecionando os artigos publicados em português, espanhol e inglês, entre os anos de 2007 e 2017, foram encontrados 81 resultados, dos quais apenas 6 foram referentes à identidade nacional brasileira, sendo que nenhum foi publicado na área de psicologia social.

$\mathrm{O}$ conceito de identidade nacional envolve, em algum sentido, o senso de comunidade política (García-García, 2015; Smith, 1991), a adesão a uma comunidade imaginada (Anderson,1991) a qual os indivíduos se sentem pertencentes (Barrett \& Davis, 2008; Lödén, 2014). Neste estudo, abordaremos este sentimento de pertença à comunidade, ao grupo nacional, e discutiremos como esta identidade está relacionada com crenças patrióticas, nacionalistas e essencialistas.

As identidades nacionais entraram em evidência após a Revolução Industrial (García-García, 1994), que possibilitou a delimitação precisa dos territórios (Sahlins, 1989) e a comunicação em massa entre os indivíduos (Gellner, 1996). Outro fator associado ao surgimento desta identidade seriam os conflitos intergrupais, pois eles podem ser considerados como um fator de coesão social, que contribue para a construção do sentimento de identidade nacional (Colley, 1992). Neste sentido, a identidade nacional surge num contexto de novas fronteiras geográficas (Nagle, 2013), quando os indivíduos de uma nação entram em contato com outras nações, o que legitimaria e daria sentido à própria identidade nacional (García-García, 1994).

Subjacente à formação da identidade nacional estaria o nacionalismo (Greenfeld \& Chirot, 1994; Hobsbawm, 2011; Leite, 2002), que funcionaria como a base ideológica na formação da identidade nacional (Tinsley, 2014; Tse, 2014). Sua principal função seria assegurar que os habitantes de uma nação se percebam como um povo especial (Hastings, 1999). O nacionalismo é uma ideologia da modernidade (Anderson, 1991; Billig, 1995; Gellner, 1996; Hobsbawm, 2011; Shulman, 2014; Smith, 2009) de difícil definição (Kunhavalik, 2009). Não obstante, para além de um movimento social, o nacionalismo pode ser considerado como um movimento ideológico que visa a autonomia, a unidade e a identidade de uma população, considerada como uma nação real ou potencial (Smith, 1991).

Diferente do nacionalismo, o patriotismo refere-se a um sentimento de lealdade ligado à nação (Sewpaul, 2009), sendo muitas vezes considerado sinônimo de nacionalismo (Billig, 1995; Sewpaul, 2009). No entanto, o patriotismo consiste no desenvolvimento de um sentimento de autopreservação, em uma moral do dever, podendo envolver inclusive o auto-sacrifício (Acton, 1985). O patriotismo está ligado ao amor à nação, porém não é sinônimo de nacionalismo. Enquanto este último faz referência à crença em uma unidade cultural e/ou étnica, o patriotismo está ligado ao amor, à forma de vida que garante a liberdade e o bem comum da nação (Viroli, 1997). Neste estudo, partimos da ideia de que o patriotismo e o nacionalismo seriam fenômenos distintos, no entanto, estariam relacionados, pois enquanto o patriotismo seria caracterizado pelo amor e lealdade à nação e pelo orgulho de sentir-se parte de uma comunidade nacional, o nacionalismo seria caracterizado por ser uma ideologia sobre a qual se sustenta a construção cultural da nação e, consequentemente, de um território e um Estado diferenciado. Essa crença pode, ademais, estar associada à ideia de superioridade de uma nação sobre as outras (Kosterman \& Feshbach, 1989).

No entanto, o nacionalismo e o patriotismo não seriam os únicos elementos associados à representação coletiva de uma nacionalidade. Os indivíduos de uma nação fazem referência à sua realidade coletiva também a partir de termos essencialistas (Pérez-Agote, 1993). De fato, os primeiros estudos sobre o caráter nacional faziam alusão a uma estrutura de personalidade que comporia a identidade coletiva dos indivíduos (Leite, 2002), partindo, portanto, da crença na existência de uma alma coletiva. O essencialismo remete à crença de que os membros de um grupo compartilham uma estrutura de personalidade, que os diferenciam dos outros grupos, o que levaria a um pensamento categórico nas relações sociais (Medin, Goldstone, \& Gentner, 1993; Yzerbyt, Rocher, \& Schadron, 1997). O essencialismo pode ser definido como um processo da categorização social caracterizado pela 
crença na existência de atributos imutáveis referentes aos entes aos quais a categorização essencialista se aplica (Pereira, Álvaro, Oliveira, \& Dantas, 2011). Trata-se, neste sentido, de um essencialismo de caráter psicológico (Estrada, Oyarzún, \& Yzerbyt, 2007).

O processo de essencialização pode ocorrer por duas vias: o naturalismo e a entitatividade. O essencialismo de caráter naturalista parte da ideia de que as características compartilhadas por um grupo têm por base leis físicas e biológicas (Estrada et al., 2007). Já o essencialismo pela via da entitatividade remete a uma avaliação dos grupos em termos de semelhança e proximidade de seus membros, que seriam vistos como indivíduos contendo um destino comum (Pereira et al., 2011), estando, portanto, mais vinculado à ideia de "nação" do que o anterior. Vale a pena ressaltar, no entanto, que tanto o primeiro como o segundo levariam à percepção de semelhanças dos membros de um determinado grupo e à generalizarização das características individuais para todo o grupo (Calquin \& Estrada, 2011; Estrada, Yzerbyt, \& Seron, 2004).

A partir do discutido até aqui, perguntamos como esses fenômenos se articulam para constituir a identidade nacional. Será que o nacionalismo, o patriotismo e o essencialismo podem ser utilizados como uma proposta explicativa da identidade nacional brasileira?

\section{Proposta de um Modelo Integrador}

A identidade nacional, na ótica da psicologia social, pode ser abordada a partir da teoria da Identidade Social. A identidade social pode ser definida como a parcela do autoconceito individual que deriva do conhecimento da pertença a um grupo, juntamente com o valor associado a esta pertença e o seu significado emocional (Tajfel, 1981). Os processos subjacentes à identidade social seriam a categorização social e a comparação social. A categorização é o processo cognitivo que se caracteriza por acentuar as diferenças aparentes, tanto físicas quanto sociais, cuja principal função seria simplificar o meio social. Nesta perspectiva, a categoria social passa a ser um grupo social. No entanto, o que dá significado a um grupo é a comparação social, definida como o processo pelo qual o indivíduo busca diferenciar-se positivamente dos outros grupos a fim de obter uma imagem positiva (Techio, Costa, Moreira, \& Hora, 2015; Torres \& Camino, 2013). É a identidade social que fornece a realidade psicológica ao grupo e que possibilita aos membros ficarem unidos em suas percepções e comportamentos coletivos (Tajfel, 1981).

A identidade vista em termos de grupos nacionais, que seria a identidade nacional, pode ser definida como a parcela do autoconceito que deriva da pertença a uma nação, juntamente com o valor associado a esta pertença e o seu significado emocional. De fato, a nação é um definidor elementar de identidade, pois é uma categoria cognitiva e emocional indispensável para explicar o mundo social (García-García, Dorado, Álvaro, \& Torres, 2016).

Por outro lado, os apegos dos indivíduos a lugares sagrados, a ícones pátrios, aos mitos que compõe a história de uma nação podem ser considerados elementos constitutivos tanto da identidade nacional (Mitra \& König, 2013; Rodríguez, 2013; Smith, 1991; Zuev \& Virchow, 2014) como do amor pátrio. Neste sentido, tanto o nacionalismo, enquanto sentimento de pertença a uma nação, como o patriotismo, enquanto sentimento de lealdade característico apenas daquelas pessoas que se sentem parte dessa nação, seriam fatores explicativos da identidade nacional. Resta-nos ainda discutir sobre o papel que o essencialismo teria num modelo explicativo da identidade nacional.

Os primeiros estudos na psicologia social referentes à nacionalidade tinham como ideia a naturalização das nações a partir dos estudos sobre o caráter nacional (Leite, 2002). O estudo da identidade nacional, a partir do ponto de vista étnico, pressupôs a crença em elementos exclusivos constituintes de tal identidade (Smith, 1991). Esta perspectiva foi abandonada por servir para disseminar os estereótipos e preconceitos que impulsionaram as guerras do nacionalismo (García-García et al., 2016).

O pensamento essencialista tem um papel fundamental no abandono do nacionalismo ét- 
nico e a substituição por um nacionalismo histórico-cutural baseado na adoção do raciocínio categórico nas relações intergrupais (Pereira et al., 2011; Yzerbyt et al., 1997). As crenças essencialistas partem da ideia de que os individuos que pertencem a uma nação compartilham características comuns e atributos semelhantes que se originam de sua pertença grupal. Desse modo, o essencialismo poderia explicar a identidade nacional por representar um processo subjacente à sua constituição. $\mathrm{O}$ essencialismo seria um mediador, por conter a heurística da categorização, pois seria o processo cognitivo que influenciaria a percepção da identidade nacional. Esta mediação seria parcial porque explicaria a parcela da identidade nacional que se refere ao processo de homogeneização baseado no conjunto de características atribuídas ao grupo nacional. Nesse sentido, hipotetizamos que as crenças essencialistas atuam como um processo cognitivo que medeiam, parcialmente, a relação entre o patriotismo, o nacionalismo e a identidade nacional (Figura 1).

Deste modo, objetivamos propor um modelo explicativo da identidade nacional a partir de crenças patrióticas, nacionalistas e essencialistas. De maneira específica, objetivamos testar a idea de que o nacionalismo e o patriotismo são elementos definidores do grau de identidade nacional e que o essencialismo tem um papel mediador na relação entre o patriotismo, o nacionalismo e a identidade nacional.

\section{Método}

\section{Participantes}

Participaram deste estudo 229 estudantes de uma universidade pública do estado da Paraíba, com idades média de 20 anos, $D P=3,6$; sendo $28,8 \%$ do sexo masculino e $71,2 \%$ do sexo feminino. Quanto à classe social, $12,2 \%$ se autoclassificaram como sendo da classe baixa, $47,6 \%$ da classe média baixa, $30,6 \%$ da classe média e $8,7 \%$ da classe média alta. No que se refere à naturalidade dos participantes $94,3 \%$ eram procedentes da região Nordeste e $3 \%$ das regiões Sudeste e Sul.

\section{Instrumentos}

O instrumento foi composto por duas partes. A primeira parte foi composta por quatro escalas, todas em formato Likert de sete pontos:

Escala de Identidade Nacional: foi operacionalizada tomando como base os estudos de Tajfel (1981) sobre identidade social, definida como o sentimento de pertença a um determinado grupo, mais a avaliação e o significado emocional dessa pertença. A escala apresentou uma solução unifatorial $(\alpha=0,87)$.

Escala de Patriotismo: retirada da escala de Kosterman e Feshback (1989), que operacionalizam o patriotismo em termos de orgulho ao país. A escala apresentou uma solução unifatorial $(\alpha=0,78)$.

Escala de Nacionalismo: operacioinalizada a partir do estudo de Terhune (1964), que concebe o nacionalismo em termos de aspirações nacionais, consideradas como consequência de uma consciência nacional. As aspirações nacionais são, deste modo, definidas como orientações compartilhadas nas quais se espera que o seu país ganhe ou extenda seu poder em relação aos outros. A escala apresentou uma solução unifatorial $(\alpha=0,75)$.

Escala de Essencialismo: retirada do estudo de Calquin e Estrada (2011) e operacionalizada em termos de crenças de que os membros de um grupo compartilham uma estrutura profunda, que os diferenciam dos outros grupos, o que levaria a um pensamento categórico nas relações sociais. A escala apresentou uma solução unifatorial $(\alpha=0,74)$.

A segunda parte foi composta por um questionário sociodemográfico contendo perguntas relativas à idade, sexo, classe social e naturalidade.

\section{Procedimentos de Coleta de Dados}

A amostragem foi não probabilística por conveniência. Os participantes foram abordados em sala de aula e solicitados a participarem da pesquisa. Foi feito um rapport informando que se tratava de um estudo a respeito de como a sociedade vê o povo brasileiro. Em seguida foi entregue aos participantes um Termo de Con- 
sentimento Livre e Esclarecido (TCLE). Após assinarem o TCLE, os participantes passaram a responder os questionários.

\section{Procedimento de Análise dos Dados}

Os dados foram analisados por meio dos softwares SPSS-20 e AMOS-18. Foram realizadas análises descritivas, correlações e regressões. As hipóteses subjacentes aos testes paramétricos foram testadas a partir de diagnósticos uni e multivariados de normalidade, multicolinearidade e presença de outliers. Para os cálculos de mediação, foram seguidos os passos propostos por Baron e Kenny (1986) e o cálculo do Intervalo de Confiança foi utilizado para verificar os efeitos indiretos dos mediadores por meio da técnica bootstrapped (Preacher \& Hayes, 2008). Neste estudo, nossa hipótese é que a identidade nacional é predita tanto pelo patriotismo quanto pelo nacionalismo e estas relações são mediadas simultaneamente pelo essencialismo (Figura 1).

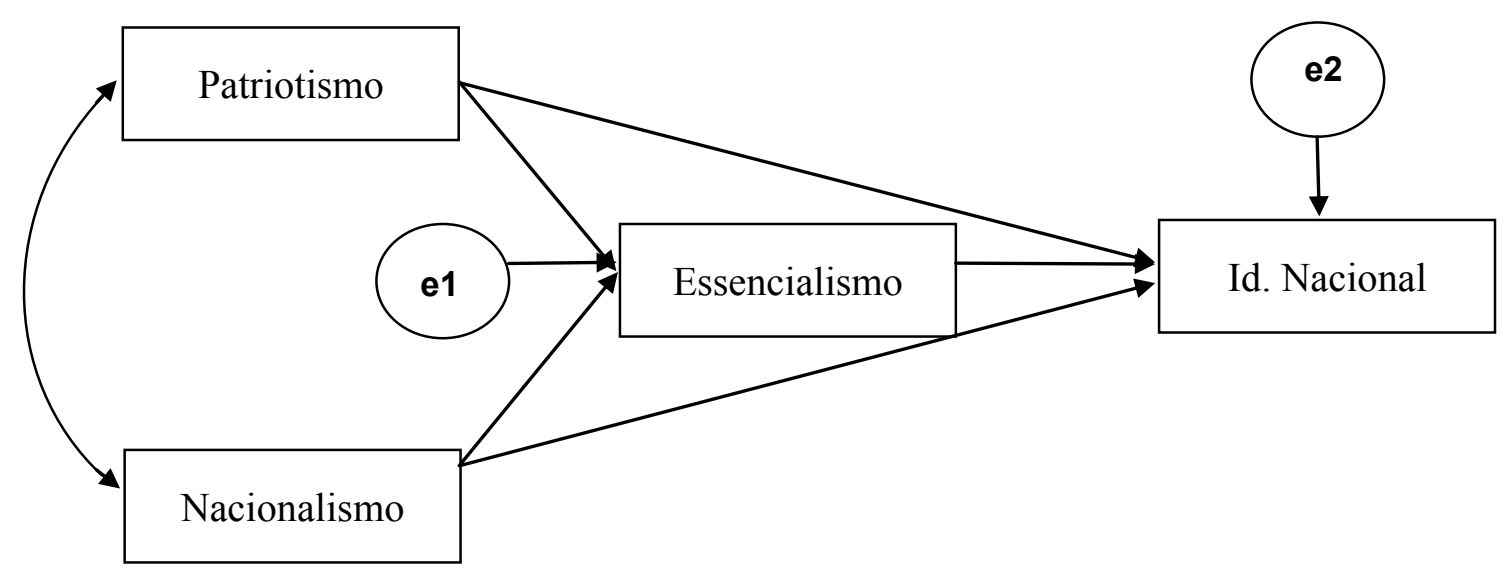

Figura 1. Modelo de Mediação da relação entre Patriotismo, Nacionalismo (VIs), essencialismo (Med) e a Identidade Nacional (VD).

\section{Procedimentos Éticos}

Após o consentimento manifesto em participar da pesquisa, os participantes assinaram um Termo de Consentimento Livre e Esclarecido, o qual esclarecia que o estudo era de caráter voluntário, que seria mantido o sigilo quanto às respostas dos participantes na publicação dos dados e que poderiam abandonar o estudo a qualquer momento. Os participantes também foram esclarecidos de que, por se tratar de uma pesquisa de opinião, não acarretaria nenhum risco; e que o estudo iria contribuir para a área da psicologia social e psicologia política, no que se refere ao tema da identidade nacional, possibilitando um melhor entendimento por parte dos brasileiros de uma das dimensões que compõe sua cidadania, a identidade nacional. Foram atendidas todas as recomendações e cuidados éticos prescritos na resolução 466/12 do Conselho
Nacional de Saúde, Protocolo 034/15. CAAE: 41019015.4.0000.5188.

\section{Resultados}

Incialmente foi realizada uma análise de correlação de Pearson, bivariada, para verificar como a identidade nacional, o patriotismo, o nacionalismo e o essencialismo estão correlacionados. Os resultados demonstraram que estas variáveis apresentam relacionamentos positivos e significativos, indicando ser possível estabelecer um modelo explicativo da identidade nacional a partir delas (Tabela 1).

Em seguida, foram realizadas análises de regressão (Tabela 2), método Enter, para satisfazer as condições subjacentes à análise de mediação (Baron \& Kenny, 1986). 
Brasileira.

Tabela 1

Análise de Correlação entre os Indicadores de Identidade Nacional, Patriotismo, Nacionalismo e Essencialismo

\begin{tabular}{|c|c|c|c|c|c|c|}
\hline & $\begin{array}{l}\text { Identidade } \\
\text { Nacional }\end{array}$ & Patriotismo & Nacionalismo & Essencialismo & \multicolumn{2}{|c|}{ Média / DP } \\
\hline Identidade Nacional & 1 & - & - & - & 5,0 & 1,2 \\
\hline Patriotismo & $0,652 * *$ & 1 & - & - & 5,0 & 1,0 \\
\hline Nacionalismo & $0,316^{* *}$ & $0,240 * *$ & 1 & - & 4,8 & 1,0 \\
\hline Essencialismo & $0,397 * *$ & $0,414 * *$ & $0,285 * *$ & 1 & 4,3 & 1,0 \\
\hline
\end{tabular}

$* * p<0,01$.

Tabela 2

Parâmetros Estimados para o Modelo de Mediação

\begin{tabular}{|c|c|c|c|c|}
\hline \multicolumn{5}{|c|}{ Variáveis Critério } \\
\hline & Passo 1 & Passo 2 & Passo 3 & Passo 4 \\
\hline & Id. Nacional & Essencialismo & Id. Nacional & Id. Nacional \\
\hline & $b$ & $b$ & $b$ & $b$ \\
\hline Intercepto & 0,397 & $1,587 * * *$ & $2,969 * * *$ & 0,169 \\
\hline Patriotismo & $0,738 * * *$ & $0,371 * * *$ & - & $0,685 * * *$ \\
\hline Nacionalismo & $0,193 * * *$ & $0,189 * * *$ & - & $0,166^{* *}$ \\
\hline \multirow[t]{5}{*}{ Essencialismo } & - & - & $0,472 * * *$ & $0,143^{*}$ \\
\hline & $R=0,673$ & $R=0,456$ & $R=0,397$ & $R=0,681$ \\
\hline & $R^{2}$ adjusted $=0,448$ & $R^{2}$ adjusted $=0,201$ & $R^{2}$ adjusted $=0,154$ & $R^{2}$ adjusted $=0,457$ \\
\hline & $F(2,226)=93,41$ & $F(2,226)=29,63$ & $F(1,227)=42,36$ & $F(3,225)=64,92$ \\
\hline & $p<0,001$ & $p<0,001$ & $p<0,001$ & $p<0,001$ \\
\hline
\end{tabular}

$* p<0,05 ; * * p<0,01 ; * * * p<0,001$

Os resultados demonstraram que tanto o patriotismo quanto o nacionalismo predizem de forma significativa a identidade nacional brasileira, satisfazendo a condição 1 para a hipótese de mediação. O patriotismo e o nacionalismo predizem de forma significativa o essencialismo, satisfazendo a condição 2 . O essencialismo apresentou efeito único e significativo sobre a identidade nacional brasileira, satisfazendo a condição 3. Por fim, verificamos que o essencialismo permanence prevendo a identidade nacional brasileira, controlando os efeitos do patriotismo e do nacionalismo, e que estas variáveis têm seus efeitos reduzidos na presença da variável mediadora, essencialismo, satisfazendo a condição 4 para que ocorra uma mediação.

Para verificar se o essencialismo, neste modelo, funciona como mediador da relação entre patriotismo, nacionalismo e identidade nacional brasileira foi executada uma análise de regressão 
por meio do software AMOS 18. A existência de outliers foi avaliada pela distância quadrada de Mahalanobis $\left(D^{2}\right)$ e a normalidade das variáveis foi avaliada pelos coeficientes de assimetria $(S k)$ e curtose $(K u)$ uni e multivariada. Nenhuma variável apresentou valores de $S k$ e $K u$ indicadores de violações à distribuição Normal $(S k<3$ e $K u<7-10$; Marôco, 2014). Nenhuma observação apresentou valores de $D^{2}$ que sugeriam a sua remoção como outliers. A multicolinearidade foi avaliada com a estatística $V I F$. Os valores de $V I F$ indicam que não há multicolinearidade entre as variáveis do modelo $(V I F s<5)$. Os efeitos indiretos que indicam se a hipótese de mediação foi satisfeita foram calculados por meio da técnica bootstrapped com 5000 reamostragens e Intervalos de Confiança ao nível de $p<0,05$.

O modelo proposto explicou $46 \%$ da variabilidade da identidade nacional brasileira. Pode-se verificar que todas as trajetórias são positivas e estatisticamente significativas. Assim, primeiramente, podemos verificar que o patrio- tismo apresentou uma relação significativa com o nacionalismo. A variável patriotismo apresentou um efeito total de 0,612 sobre a identidade nacional brasileira, com efeito direto de 0,568 e efeito indireto, mediado pelo essencialismo, de 0,044 . Já a variável nacionalismo apresentou um efeito total de 0,170 sobre a identidade nacional brasileira, com efeito direto de $0,146 \mathrm{e}$ efeito indireto, mediado pelo essencialismo, de 0,024 . De acordo com a técnica de reamostragem bootstrapped, o efeito indireto $\left(\mathrm{c}^{1}=0,044\right)$ foi significativo ao nível $95 \%$ com IC variando entre 0,001 e $0,047, p<0,05$. O efeito indireto $\left(c^{2}\right.$ $=0,024)$ também foi significativo ao nível $95 \%$ com IC variando entre 0,002 e $0,030, p<0,05$. Deste modo, verificamos que a relação entre o patriotismo, o nacionalismo e a identidade nacional brasileira foi mediada parcialmente pelo essencialismo. A Figura 2 apresenta o modelo com as estimativas estandardizadas dos coeficientes de regressão e o $R^{2}$ da identidade nacional brasileira.

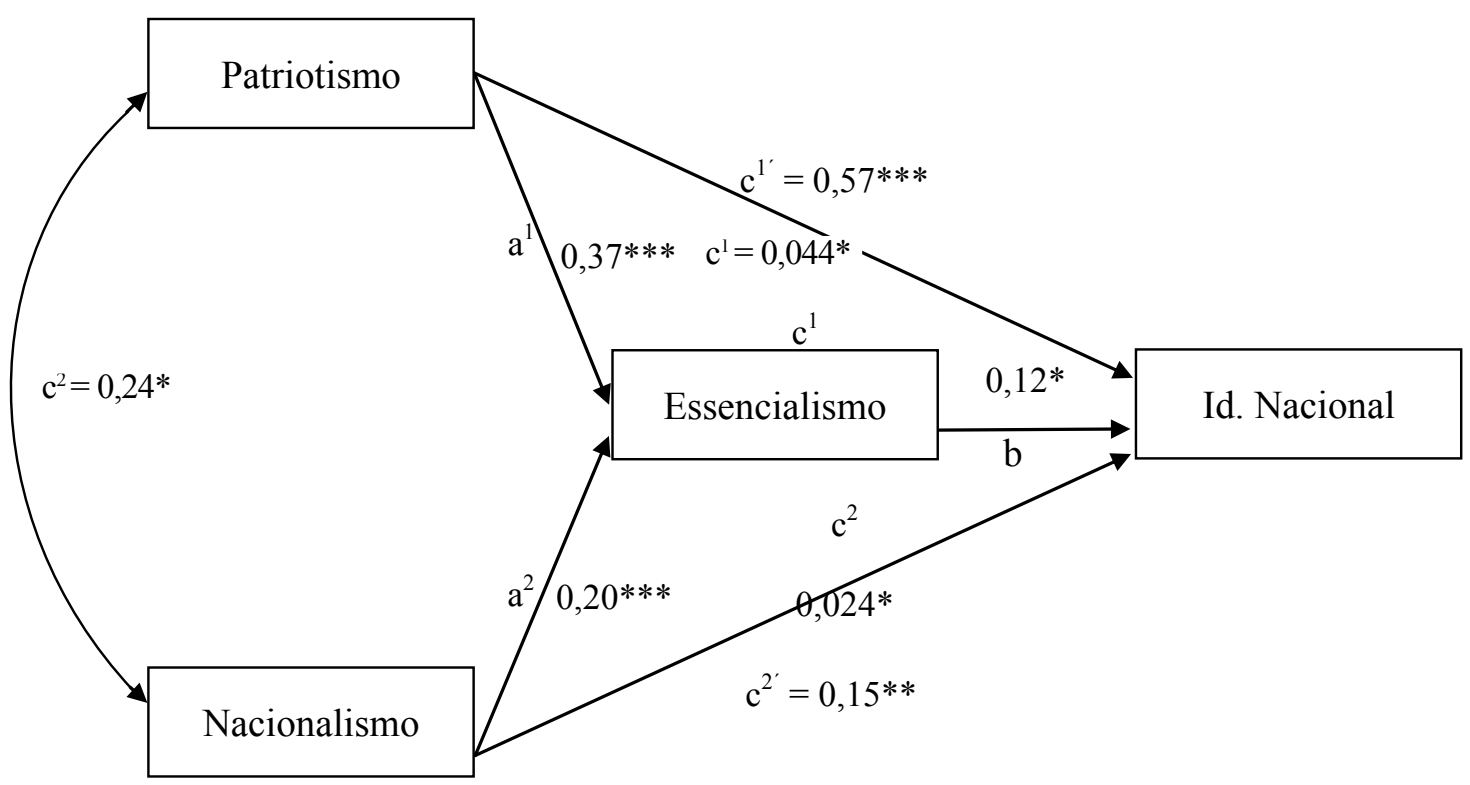

Figura 2. Modelo de Mediação da relação entre Patriotismo, Nacionalismo (VIs), Essencialismo (Med) e a Identidade Nacional (VD). $* p<0,05 ; * * p<0,01 ; * * * \mathrm{p}<0,001$.

Estes resultados demonstram, num primeiro momento, que a identidade nacional brasileira é um construto multifacetado e que pode ser explicada por recurso a dimensões como patriotismo, nacionalismo e essencialismo. De forma mais específica, a Figura 2 demonstra os papeis específicos destas variáveis no modelo de regressão. Tanto o patriotismo quanto o nacionalismo são 
construtos que constituem a identidade nacional. Neste sentido, quanto mais os indivíduos aderem a crenças patrióticas e nacionalistas maior é a probabilidade de apresentarem altos níveis de identidade nacional.

O essencialismo, por outro lado, desempenhou um papel distinto, pois funcionou como um tipo de crença, de natureza categórica, que é ativada ao se pensar em termos patrióticos e nacionalistas. Deste modo, quando os indivíduos pensam em suas identidades nacionais, o fazem, em parte, a partir de crenças essencialistas, caracterizadas, neste estudo, pela crença na existência de um conjunto de atributos inerentes ao grupo dos brasileiros. Isto não quer dizer, no entanto, que há um componente essencialista constitutivo da identidade nacional brasileira, mas que a categorização social, processo subjacente ao pensamento essencialista, influencia a maneira como os indivíduos percebem sua nacionalidade.

\section{Discussão}

Este estudo objetivou propor um modelo explicativo da identidade nacional a partir de crenças patrióticas, nacionalistas e essencialistas. Especificamente, objetivamos testar a hipótese de que o essencialismo tem um papel mediador na relação entre o patriotismo, o nacionalismo e a identidade nacional. Os resultados corroboraram nossa hipótese, no sentido de que o essencialismo mediou parcialmente estas relações. Isto significa que mesmo os indivíduos aderindo a crenças essencialistas, o patriotismo e o nacionalismo ainda permanecem atuando como variáveis explicativas no modelo.

De fato, tanto o nacionalismo quanto o patriotismo são construtos formadores da identidade nacional (Greenfeld \& Chirot, 1994; Hobsbawm, 2011; Kosterman \& Feshbach, 1989; Lee, 2014; Piwoni, 2015; Tse, 2014). Neste modelo pode-se observar que ambos apresentam baixa correlação entre si (Ariely, 2012; Gries, Zhang, Crowson, \& Cai, 2011) e alto poder preditivo da identidade nacional (Miller-Idriss \& Rothenberg, 2011; Pérez-Agote, 1993; Quiroga, 2014; Tartakovisky, 2011), indicando que são fenômenos distintos (Kosterman \& Feshbach, 1989; Viroli, 1997).

No que se refere ao essencialismo, consideramos no nosso modelo que esta variável não possui um papel constitutivo da nacionalidade, mas sim processual. Seria ele quem explicaria a parcela da identidade nacional percebida em termos de atributos homogêneos (Smith, 1991), inerentes ao grupo nacional. Esta percepção passa a ter significado no processo de comparação com outras nações (Colley, 1992; Sahlins, 1989), o que legitima e dá sentido à identidade nacional (García-García, 1994). Isto seria desta maneira porque subjacente ao pensamento essencialista estaria a heurística da categorização social (Pereira et al., 2011; Calquin \& Estrada, 2011; Estrada et al., 2007; Estrada et al., 2004; Pereira, Álvaro, \& Garrido, 2016), que simplifica a realidade ao selecionar um conjunto limitado de atributos para representar os grupos sociais, neste caso a nação (Laxer, Carson, \& Korteweg, 2014). Como a forma com que os indivíduos se relacionam com os grupos sociais é semelhante à maneira pela qual se relacionam com sua nação de pertença (Terhune, 1964; Troch, 2013), o essencialismo também estaria funcionando ao simplificar a complexidade do fenômeno "identidade nacional", organizando-a de forma categórica (Patel, 2013; Raqib \& Barreto, 2014). Uma vez que a categorização social é um processo em comum ao pensamento essencialista e à identidade social nacional, os conteúdos constitutivos das crenças essencialistas associados aos grupos nacionais influenciariam a maneira pela qual a identidade é percebida. De maneira geral, de acordo com o modelo proposto, o essencialismo não constitui a identidade nacional, mas funciona como uma heurística através da qual os indivíduos organizam as informações relativas aos grupos nacionais por meios dos processos de simplificação do meio social, permitindo uma maior sensação de controle psicológico (Estrada et al., 2007; Estrada et al., 2004), e da categorização social, por meio da qual os indivíduos ancoram e dão significado as suas experiências (Moscovici, 2015).

Por se tratar de uma nova proposta explicativa da identidade nacional, ainda não é possível 
generalizar estes resultados para toda a população. $\mathrm{O}$ fato da amostra ter sido composta por estudantes universitários também se apresenta como um limite deste estudo e aponta para outras possibilidades, com diferentes participantes, o que possibilita testar, em diferentes contextos, este modelo. $\mathrm{O}$ modelo proposto não pretende abarcar toda a complexidade da identidade nacional (Sim \& Leith, 2014; Sperling, 2013, Storm, 2015; Zwet, 2015), mas tem como principal objetivo tentar compreender como as variáveis, neste caso o patriotismo, o nacionalismo e o essencialismo, se configuram para explicar esta identidade.

\section{Considerações Finais}

A psicologia social brasileira tem dado pouca importância ao estudo da identidade nacional, mesmo sendo um dos fenômenos de maior impacto político dos nossos tempos (Anderson, 1991). Na tentativa de preencher esta lacuna empírica e dar um passo a frente nos estudos sobre a identidade nacional, procuramos apresentar um novo modelo explicativo desta identidade, articulando variáveis do âmbito cognitivo e psicossocial.

Ao pensar a identidade nacional a partir de um modelo em que o essencialismo exerce um papel processual, passamos a entender, em certa medida, o porquê que os teóricos do caráter nacional terem adotado uma perspectiva essencialista (Leite, 2002; Mead, 1951) e porquê ela tem estado associada à formação de estereótipos e preconceitos nacionais (García-García et al., 2016). Deste modo, ao constatar que a maneira que o essencialismo explica a identidade nacional é distinta dos estudos tradicionais, trazemos de volta mais uma vez os questionamentos de como esta identidade é constituída e quais os demais processos subjacentes a esta formação.

Uma vez que estes resultados são referentes a uma parcela da população, a saber estudantes universitários, e que o desenho do estudo é correlacional, ainda não é possível fazer inferências para o conjunto da população brasileira. Estudos com amostras populacionais, representando as demais regiões do país, podem dar uma maior sustentação ao modelo proposto, bem como acrescentar novas perspectivas relativas à construção da identidade nacional (Leal, 2014), de modo a esclarecer um pouco mais o fenômeno da identidade nacional e poder visualizar suas principais consequências sociais.

\section{Contribuições dos Autores}

Contribuição substancial no conceito e desenho do estudo: Eldo Lima Leite; Ana Raquel Rosas Torres; José Luiz Álvaro Estramiana.

Contribuição na coleta de dados: José Roniere Morais Batista; Andreza Silene Silva Ferreira.

Contribuição na análise e interpretação de dados: Eldo Lima Leite; Andreza Silene Silva Ferreira; Ana Raquel Rosas Torres; José Luiz Álvaro Estramiana; José Roniere Morais Batista.

Contribuição para a preparação do manuscrito: Eldo Lima Leite; Andreza Silene Silva Ferreira; Ana Raquel Rosas Torres.

Contribuição à revisão crítica, agregando conteúdo intelectual: José Luiz Álvaro Estramiana; Ana Raquel Rosas Torres.

\section{Conflitos de interesse}

Os autores declaram não ter conflito de interesse relacionado à publicação deste manuscrito.

\section{Referências}

Acton, J. E. E. D. (1985). Selected writings of Lord Acton. In J. Rufus Fears (Ed.), Essays in the history of liberty: Vol 1. Indianapolis, IN: Liberty Fund.

Anderson, B. (1991). Imagined Communities. Reflectinos on the Origen and spread of Nationalism. London: Verson.

Ariely, G. (2012). Globalisation and the decline of national identity? An exploration across sixty-three countries. Nations and Nationalism, 18(3), 461482. doi: 10.1111/j.1469-8129.2011.00532.x

Baron, R. M., \& Kenny, D. A. (1986). The moderator-mediator variable distinction in social psychological research: Conceptual, strategic, and statistical considerations. Journal of Personality 
and Social Psychology, 51(6), 1173-1182. doi: https://doi.org/10.1037//0022-3514.51.6.1173

Barrett, M., \& Davis, S. C. (2008). Applying social identity and self-categorization theories to children's racial, ethnic, national and state identifications and attitudes. In S. M. Quintana \& C. McKown (Eds.), Handbook of race, racism and the developing child (pp. 72-110). Hoboken, NJ: Wiley.

Billig, M. (1995). Banal Nationalism. London: Sage.

Calquin, D., \& Estrada, C. (2011). Esencialismo, su impacto en la percepción de similitud y el uso de diferente tipo de información en la formación de impresión. Psykhe, 20(1), 63-75. doi: http:// dx.doi.org/10.4067/S0718-22282011000100005

Cavusgil, S. T., \& Kardes, I. (2013). Brazil: Rapid development, internationalization, and middle class formation. Revista Eletrônica de Negócios Internacionais, 8(1), 1-16. doi: http://dx.doi. org/10.18568/1980-4865.811-16

Colley, L. (1992). Britons: Forging the Nation 1707 1837. London: Yale University Press.

Estrada, C., Oyarzún, M., \& Yzerbyt, V. (2007). Implicit Theories and Psychological Essentialism: Conceptual tools for the study of the relations between and within the groups. Psykhe, 16(1), 111121. doi: $10.4067 / \mathrm{S} 0718-22282007000100009$

Estrada, C., Yzerbyt, V., \& Seron, E. (2004). Efecto del esencialismo psicológico sobre las teorías ingênuas de las diferencias grupales. Psicothema. 16(2), 181-186. Recuperado em http://www.redalyc.org/html/727/72716202/

García-García, J. (1994). Nacion, identidad y paradoja: una perspectiva relacional del nacionalismo. Revista Internacional de Investigaciones Sociológicas, 64, 165-183. doi: 10.2307/40183739

García-García, J. (2015). Nación, sujeto y psique: la construcción psicológica del nacionalismo. Athenea Digital, 15(1), 333-346. doi: http:// dx.doi.org/10.5565/rev/athenea.1606

García-García, J., Dorado, S. R., Álvaro, J. L., \& Torres, A. R. R. (2016). Nacionalismo e identidade nacional sob uma perspectiva psicossociológica. In M. E. O. Lima, A. R. R. Torres, \& E. M. Techio (Eds.), Identidade nacional e representações do Brasil: Abordagens integrativas (pp. 27-54). São Paulo, SP: Scortecci.

Gellner, E. (1996). O advento do nacionalismo e sua interpretação: Os mitos da nação e da classe. In G. Balakrishnan (Ed.), Um Mapa da Ques- tão Nacional (pp. 107-154). Rio de Janeiro, RJ: Contraponto.

Greenfeld, L., \& Chirot, D. T. S. (1994). Natinalism and Agression. Theory and Society, 23(1), 79130. doi: https://doi.org/10.1007/BF00993674

Gries, P. H., Zhang, H. Q., Crowson, M., \& Cai, H. (2011). Patriotism, Nationalism and China's US Policy: Structures and consequences of Chinese National Identity. The China Quarterly, 205, 1-17. doi: 10.1017/S0305741010001360

Hastings, A. (1999). Special peoples. Nations and Nationalism. 5(3), 381-396. doi: 10.1111/j.1354$-5078.1999 .00381 . x$

Hobsbawm, E. J. (2011). Nações e Nacinalismo desde 1780: Programa, mito e realidade. (Edição Esp., Vol. 18). Rio de Janeiro, RJ: Saraiva.

Kosterman, R., \& Feshbach, S. (1989). Toward a measure of patriotic and nationalistic atititudes. Poltical Psychology, 10(2), 257-274. doi: http:// dx.doi.org/10.2307/3791647

Kunhavalik, J. P. (2009). Os militares e o conceito de nacionalismo: Disputas retóricas na década de 1950 e início dos anos 1960. Santa Catarina, SC: Universidade Federal de Santa Catarina.

Laxer, E., Carson, R. D., \& Kortebeg, A. C. (2014). Articulating minority nationhood: Cultural and political dimensions in Québec's reasonable accommodation debate. Nations and Nationalism 20(1), 133-153. doi: 10.1111/nana.12046

Leal, J. (2014). What's (not) in a parade? Nationhood, ethnicity and regionalism in a diasporic context. Nations and Nationalism 20(2), 200-217. doi: 10.1111/nana.12062

Lee, J. W. (2014). Legacies of Japanese colonialism in the rhetorical constitution of South Korean national identity. National Identities, 16(1), 1-13. doi: http://dx.doi.org/10.1080/14608944.2 013.843516

Leite, D. M. (2002). O Caráter Nacional Brasileiro. São Paulo, SP: Livraria Pioneira.

Lima, M. E. O., Torres, A. R. R., \& Techio, E. M. (2016). Introdução. In M. E. O. Lima, A. R. R. Torres, \& E. M. Techio (Eds.), Identidade nacional e representações do Brasil: Abordagens integrativas (pp. 21-26). São Paulo, SP: Scortecci.

Lödén, H. (2014). Peace, love, depoliticisation and the domestic alien: National identity in the memorial messages collected after the terror attacks in Norway 22 July 2011. National Identities, 
16(2), 157-176. doi: http://dx.doi.org/10.1080/1 4608944.2014 .918593

Marôco, J. (2014). Análise de Equações Estruturais: Fundamentos Teóricos, Softwares e Aplicações. Pêro Pinheiro, Portugal: Cafilesa.

Mead, M. (1951). The study of national character. In D. Lerner \& H. K. Lasswell (Eds.), The Policy Science (pp.70-85). Stanford, UK: Stanford University Press.

Medin, D., Goldstone, R., \& Gentner, D. (1993). Respects for similarity. Psychological Review, 100(2), 254-278. doi: http://dx.doi. org/10.1037/0033-295X.100.2.254

Miller-Idriss, C., \& Rothenbeg, B. (2011). Ambivalence, pride and shame: Conceptualisations of German nationhood. Nations and Nationalism 18(1), 132-135. doi: 10.1111/j.14698129.2011.00498.x

Mitra, S. K., \& König, L. (2013). Icon-ising national identity: France and India in comparative perspective. National Identities, 15(4), 357-377. doi: http://dx.doi.org/10.1080/14608944.2013.8 29430

Moscovici, S. (2015). Representações Sociais: Investigações em psicologia social. Petrópolis, RJ: Vozes.

Nagle, S. (2013). Confessional identity as national boundary in national historical narratives: Ireland and Germany compared. Studies in Ethnicity and Nationalism, 13(1), 38-56. doi: 10.1111/sena.12015

Patel, K. K. (2013). Where and when was Europe? Europeanness and its relationship to migration. National Identities, 15(1), 21-32. doi: http:// dx.doi.org/10.1080/14608944.2012.733152

Pereira, M. E., Álvaro, J. L., \& Garrido, A. (2016). Essentialization processes in men 45 and women: A Brazil-Spain comparative study. Anales de Psicología, 32(1), 190-198.

Pereira, M. E., Álvaro, J. L., Oliveira, A. C., \& Dantas, G. S. (2011). Estereótipos e essencialização de brancos e negros: Um estudo comparativo. Psicologia \& Sociedade, 23(1), 144-153. Recuperado em http://www.scielo.br/pdf/psoc/ v23n1/a16v23n1

Pérez-Agote, A. (1993). Las Paradojas de La Nacion. Reis: Revista Española de Investigaciones Sociológicas, 61, 7-22. doi: 10.2307/40183615
Piwoni, E. (2015). Claiming the nation for the people: The dynamics of representation in German public discourse about immigrant integration. Nations and Nationalism, 21(1), 83-101. doi: 10.1111/nana.12084

Preacher, K. J., \& Hayes, A. F. (2008). Asymptotic and resampling strategies for assessing and comparing indirect effects in multiple mediator models. Behavior Research Methods, 40(3), 879891. doi: https://doi.org/10.3758/BRM.40.3.879

Quiroga, A. (2014). The three spheres. A theoretical model of mass nationalisation: The case of Spain. Nations and Nationalism, 20(4), 683700. doi: 10.1111/nana.12073

Raqib, M., \& Barreto, A. A. (2014). The Taliban, religious revival and innovation in Afghan nationalism. National Identities, 16(1), 15-30. doi: http://dx.doi.org/10.1080/14608944.2013.8 43517

Reicher, M., \& Ropkins, N. (2001). Self and nation. London: Sage.

Rodríguez, F. M. (2013). Representing the nation: Art and identity in Porfirian Mexico. National Identities, 15(4), 333-355. doi: http://dx.doi.org/ $10.1080 / 14608944.2013 .811225$

Sahlins, P. (1989). Boundaries. The Making of France and Spain in the Pyrenees. Berkeley, CA: University of California Press.

Sewpaul, V. (2009). On national identity, nationalism and Soccer 2010: Should social work be concerned? International Social Work, 52(2), 143-153.

Shulman, S. (2014). Nation versus class in Ukraine. Nations and Nationalism. 20(1), 154-171. doi: 10.1111/nana. 12056

Sim, D., \& Leith, M. S. (2014). Scottish diasporic identities in the Netherlands. National Identities, 16(2), 139-155. doi: http://dx.doi.org/10.1080/1 4608944.2014.909799

Smith, A. D. (1991). National Identity. London: University of Nevada Press.

Smith, A. D. (2009). Ethno-symbolism and nationalism: A cultural approach. London: Routledge.

Sobral, J. M., \& Vala, J. (2010). Introdução. In J. M. Sobral \& J. Vala (Eds.), Identidade nacional, inclusão e exclusão social (pp. 17-29). Lisboa: Instituto de Ciências Sociais da Universidade de Lisboa. 
Sperling, J. (2013). Belonging, beyond the nation: The significance and meaning of European identity for Latin American-origin youth in Spain. National Identities, 15(1), 67-84. doi: http:// dx.doi.org/10.1080/14608944.2012.733155

Storm, E. (2015). Nationalism studies between methodological nationalism and orientalism: An alternative approach illustrated with the case of El Greco in Toledo, Spain. Nations and Nationalism, 21(4), 786-804. doi: 10.1111/nana.12111

Tajfel, H. (1981). Human groups and social categories. Studies in social psychology. Cambridge, MA: Cambridge University Press.

Tartakovisky, E. (2011). National identity of highschool adolescents in an Era of Socio-Economic Change: Russia and Ukraine in the Post-Perestroika. Journal of Youth and Adolescence, 40(2), 231-244. doi: 10.1007/s10964-010-9509. 6

Techio, E. M., Costa, P. N., Moreira, T. A., \& Hora, N. (2015). Identidade social baiana: Ser baiano na concepção de universitários. Revista Brasileira de Psicologia, 2(1), 79-89.

Terhune, K. W. (1964). Nationalism among foreign and American students: An exploratory study. The Journal of Conflict Resolution, 8(3), 256-270. doi: https://doi. org/10.1177/002200276400800304

Terlouw, K. (2013). Performing identities on a Dutch River Dike: National identity and diverging lifestyles. Studies in Ethnicity and Nationalism, 13(2), 233-255. doi: 10.1111/sena.12035

Tinsley, M. (2014). 'We Will Re-Member Them': Muslims in the Great War Semi-Centenary. Studies in Ethnicity and Nationalism, 14(3), 399-417. doi: 10.1111/sena.12101

Torres, A. R. R., \& Camino, L. (2013). Grupos sociais, relações intergrupais e identidade social. In L. Camino, A. R. R. Torres, M. E. O.
Lima, \& M. E. Pereira (Eds.), Psicologia Social: Temas e Teorias (pp. 515-539). Brasília, DF: Tecknopolitik.

Troch, P. (2013). Interactive nationhood: The relation between Croatian and Yugoslav national identity in the interwar period. Nations and Nationalism 19(4), 781-798. doi: 10.1111/nana.12045

Tse, T. K. (2014). Constructing Chinese identity in Post-colonial Hong Kong: A discursive analysis of the Official Nation-Building Project. Studies in Ethnicity and Nationalism, 14(1), 188-206. doi: 10.1111/sena.12073

Viroli, M. (1997). For Love of Country: An essay on patriostism and nationalism. Oxford, UK: Oxford University. doi: 10.1093/0198293585.001.0001

Yzerbyt, V., Rocher, S., \& Schadron, G. (1997). Stereotypes as explanations: A subjective essentialistic view of group perception. In R. Spears, P. J. Oakes, N. Ellemers, \& S. A. Haslam (Eds.), The social psychology of stereotyping and group life (pp. 20-50). Malden, MA: Blackwell.

Zuev, D., \& Virchow, F. (2014). Performing nationalidentity: The many logics of producing national belongings in public rituals and events. Nations and Nationalism, 20(2), 191-199. doi: 10.1111/ nana.12063

Zwet, A. (2015). Operationalising national identity: The cases of the Scottish National Party and Frisian National Party. Nations and Nationalism, 21(1), 62-82. doi: 10.1111/nana.12091

(cc) BY (c) O(s) autor(es), 2018. Acesso aberto. Este artigo está distribuído nos termos da Licença Internacional Creative Commons Atribuição 4.0 (http://creativecommons.org/licenses/by/4.0/), que permite o uso, distribuição e reprodução sem restrições em qualquer meio, desde que você dê crédito apropriado ao(s) autor(es) original(ais) e à fonte, fornecer um link para a licença Creative Commons e indicar se as alterações foram feitas. 